\title{
Rapid and Sensitive Detection of Bisphenol A from Serum Matrix
}

Xiaogang Lin ${ }^{1,2}$, Cheng Cheng ${ }^{2}$, Paul Terry ${ }^{3}$, Jiangang Chen ${ }^{4, \#}$, Haochen $\mathrm{Cui}^{2}$, Jayne $\mathrm{Wu}^{2,{ }^{*}}$

1. Key Laboratory of Optoelectronic Technology and System of the Education Ministry of China, Chongqing University, Chongqing 400044, China

2. Department of Electrical Engineering and Computer Science, the University of Tennessee, Knoxville, TN 37996, USA

3. Department of Medicine, the University of Tennessee Graduate School of Medical Center

4. Department of Public Health, the University of Tennessee, Knoxville, TN 37996, USA

* corresponding author, jwu10@utk.edu

\# co-corresponding author, jchen38@utk.edu

\begin{abstract}
:
Bisphenol A (BPA) is an endocrine disrupting compound that may have adverse developmental, reproductive, neurological, and immune system effects. Low-level exposure to BPA is ubiquitous in human populations due to its widespread use in consumer products. Therefore, highly sensitive methods are needed to quantify BPA in various matrices including water, serum, and food products. In this study, we developed a simple, rapid, highly sensitive and specific sensor based on an aptamer probe and AC electrokinetics capacitive sensing method that successfully detected BPA at femto molar (fM) levels, which is an improvement over prior work by a factor of 10 . We were able to detect BPA spiked in human serum as well as in maternal and cord blood within 30 seconds. The sensor is responsive to BPA down to femto molar levels, but not to structurally similar compounds including bisphenol F (BPF) or bisphenol S (BPS) even at much higher concentration. Further development of this platform may prove useful in monitoring exposure to BPA and other small molecules in various matrices.
\end{abstract}

Key words: AC electrokinetics; BPA, affinity sensor; capacitive biosensor. 


\section{Introduction}

Bisphenol A (BPA) is a key monomer in the production of the most common form of clear and shatter-proof polycarbonate plastic. Low-level exposure to BPA is ubiquitous in human populations due to its widespread use in consumer products. Evidence from in vitro studies and in vivo animal models (Chen et al., 2015; Suvorov et al., 2015) suggests that BPA's underlying endocrine disrupting mechanisms are diverse, and may adversely impact endogenous hormone biosynthesis, metabolism, and homeostatic balance (Yoon et al., 2014; Fenichel et al., 2013).

A simple and affordable method to detect low levels of BPA in environmental and biological samples would facilitate the evaluation of environmental contamination and the various health risks associated with endocrine disruption. Due to wide use of BPA in consumer products, BPA has been detected in biological fluids of humans who have had no known occupational exposure (He et al., 2009). Conventional BPA detection methods include liquid chromatography coupled with mass spectrometry (LC-MS) (Inoue et al., 2000), liquid chromatography with electrochemical detection (LC-ED) (D'Antuono et al., 2001), and gas chromatography coupled with mass spectrometry (GC/MS) (Meesters et al., 2003). These methods are time-consuming and require expensive instruments operated by highly skilled personnel. Recently, several biosensing approaches have been investigated for BPA detection based on their high sensitivity and relatively low operational cost. (Ragavan et al., 2013). These approaches include an enzyme-linked immunosorbent assay (ELISA), a surface plasmon resonance sensor, and various types of electrochemical sensors. Among the latter technologies, electrochemical sensing may be most applicable for rapid BPA detection due to low cost, ease of use, and high throughput capacity (Hegnerová et al., 2010; Sajiki et al., 2008; Kim et al., 2007). Current research with electrochemical sensing focuses on emerging sensing materials. Materials including molecularly-imprinted polymers (Avila et al., 2008), carbon-nanotube (Yang et al., 2010; Vashist et al., 2011), graphene (Ntsendwana et al., 2012; Ndlovu et al., 2012), quantum dots (Yin et al., 2010), nanocomposites (Yin et al., 2009) and metal composites (Nambiar et al., 2011)) have been developed to increase the surface area of electrodes to enhance current signals from redox reactions to achieve small molecule detection. 
In this study, we developed a simple, rapid, highly sensitive and specific BPA sensor based on an aptamer probe and an AC electrokinetics (ACEK) capacitive sensing method (Cui et al., 2013; Li et al., 2014; Rocha et al., 2016; Cheng et al., 2016). Aptamers are synthetic nucleic acid molecules that can bind to non-nucleic acid targets (e.g., small molecules, proteins, and cells) with high specificity and affinity. They are obtained through an in vitro selection process known as systematic evolution of ligands by exponential enrichment (SELEX). Aptamer, as a probe for affinity assay, has several advantages. It is small in size, which is essential for a capacitive biosensor. It is highly stable and flexible under different chemical testing environments, making it adaptable to sample solutions with varying $\mathrm{pH}$, temperatures and ionic strengths, which may be stringent for methods such as antibody based assays (Walter et al., 2012). This characteristic of aptamers is important because detection was done with water as the background solution, as opposed to commonly used phosphate-buffered saline (PBS). When using the ACEK capacitive sensing method, an AC signal at a fixed frequency is applied over an array of planar microelectrode sensors in sample fluids. The AC signal induces ACEK microflows to promote probe-analyte binding and at the same time directly reads the interfacial capacitance change caused by molecular binding at the interface of sensor electrode and sample fluid. Our prior work using ACEK capacitive sensing demonstrated a BPA-specific aptasensor modified from a commercially available microelectrode array (Cui et al., 2015). In the present work, we used in-house microfabricated interdigitated electrodes as the sensor, with a characteristic length of 6 microns versus the 1.4 microns used in previous work. The change in sensor design is based on the finding that larger microelectrodes yield stronger ACEK microflows for in-site enrichment of analyte molecules (Yuan et al., 2014, Cui et al., 2016); BPA enrichment is predominated achieved through ACEK microflows.

With the improved sensor design and use of an aptamer probe, we successfully detected BPA at femto molar level in biological fluids. This sensor also demonstrated good specificity, such that it was not responsive to 4,4'Methylenediphenol (bisphenol F, BPF) or 4,4'-Sulfonyldiphenol (bisphenol S, BPS), which are common BPA substitutes. The further development of this sensing method may offer valuable opportunities for the detection of other small environmental molecules. 


\section{Sensing and enrichment mechanisms}

2.1 Interfacial capacitance sensing mechanisms

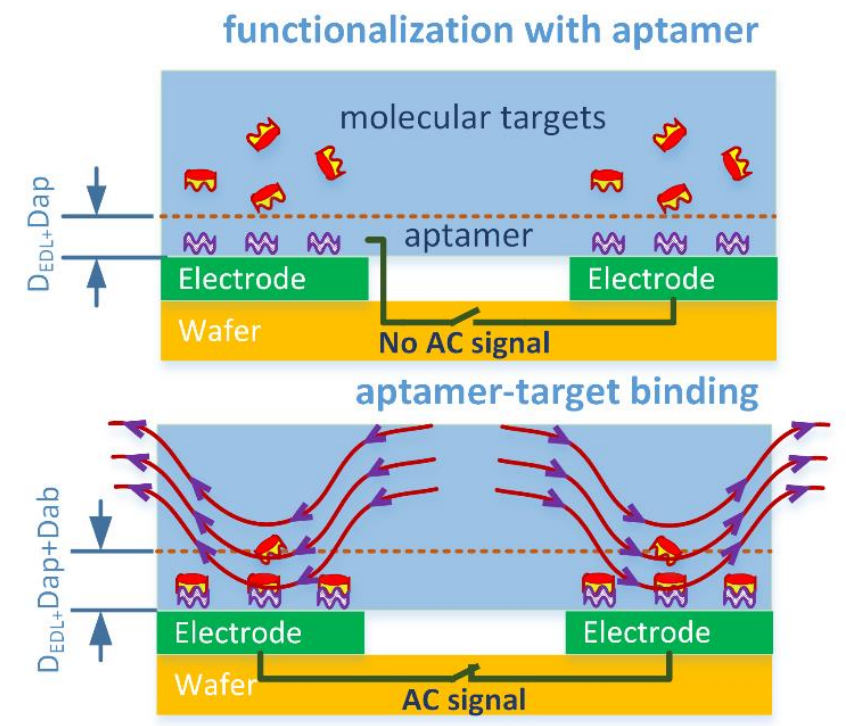

Fig. 1. Changes at the electrode surface due to the binding of specific target to immobilized aptamer

When a pair of interdigitated microelectrodes is immersed in electrolyte, the electrode impedance can be approximated as a network of capacitors and resistors, including interfacial capacitors, charge transfer resistance, and electrolyte resistance, as conceptually shown in Fig. 1. Electrical double layer (EDL) is a structure that appears on the surface of a microelectrode when it is exposed to a fluid. Electrically, EDL can be modeled as a capacitor. The layers of counter ions and surface charges are equivalent to the two plates in a capacitor, and the plate separation distance is the EDL thickness. When aptamer molecules are adsorbed onto the electrode surface, the interfacial capacitance $C_{i n t}$ will change due to the change in the thickness and surface area of $C_{i n t}$, which can then be used to indicate the deposition of aptamer molecules on the electrode.

Before the affinity assay, the electrode surface is immobilized with a layer of aptamer molecules to achieve specificity for the targeted particle. The interfacial capacitance can be approximated as

$$
C_{i n t}=\frac{A_{i n t}}{\left(\frac{D_{a p}}{\varepsilon_{p}}+\frac{D_{E D L}}{\varepsilon_{S}}\right)}
$$


where $\varepsilon_{s}$ and $\varepsilon_{p}$ are the permittivities of the EDL and the aptamer. $A_{\text {int }}$ is the electrode area and $D_{a p}$ is the aptamer thickness. $D_{E D L}$ is the electrical double layer (EDL) thickness.

During sensing experiments, sample solution containing the target molecule are applied over the functionalized electrodes. When the aptamer begins to capture the molecular targets, the molecular deposition on the sensor surface becomes thicker, and the interfacial capacitance $C_{i n t}$ is expected to change to

$$
C_{\text {int }, \text { binding }}=\frac{A_{\text {int }, \text { binding }}}{\left(\frac{D_{a b}}{\varepsilon_{t}}+\frac{D_{a p}}{\varepsilon_{p}}+\frac{D_{E D L}}{\varepsilon_{S}}\right)}
$$

where $\varepsilon_{t}$ is the permittivity of the target particle, $D_{a b}$ is the bounded molecular targets thickness, and $A_{\text {int,binding }}$ is the effective area of the interfacial capacitor (Assuming the electrode area remains the same before and after the binding $A_{\text {int }}=$ $A_{\text {int,binding. }}$ ) From formula (2), it follows that the change in the thickness of the dielectric layer will cause a decrease in the interfacial capacitance. In this work, the capacitive biosensing utilizes the change in $C_{i n t}$. Furthermore, the normalized capacitance change rate is used to indicate aptamer binding events. The normalized change in capacitance is

$$
\Delta C / C_{\text {int }}=\left(C_{\text {int }, \text { binding }}-C_{\text {int }}\right) / C_{\text {int }}=-D_{a b} /\left(D_{a b}+\frac{\varepsilon_{t}}{\varepsilon_{p}} D_{a p}+\frac{\varepsilon_{t}}{\varepsilon_{s}} D_{D E L}\right)
$$

Consequently, $\Delta C / C_{\text {int }}$ can be directly correlated with the amount of bounded macromolecules on the electrode surface; this is a quantitative detection method. Also, using the normalized capacitance change rate, i.e. $\Delta C / C_{i n t}$, helps to improve the test repeatability because it is independent of the sensor area.

\subsection{ACEK enrichment mechanism}

ACEK is an effective method for in-situ analyte enrichment (Wu, 2008). An $\mathrm{AC}$ electric field can generate microflows through AC electroosmosis (ACEO) (Wu et al., 2005, Hart et al., 2010) and/or an AC electrothermal (ACET) effect (Lian et al., 2008, Yang et al., 2010) to carry bioparticles to the sensor, or directly exert force on an uncharged particle through a mechanism known as dielectrophoresis (DEP). Each of the three mechanisms (DEP, ACEO and ACET effects) has been used for accelerated binding and detection. 
DEP was utilized for enrichment and rapid detection of antibody and bacteria in prior work ( $\mathrm{Li}$ et al 2015, Rocha et al 2016). The DEP velocity of a spherical particle is expressed as (Castellanos et al., 2010):

$$
\left\langle u_{D E P}\right\rangle=\frac{a^{2} \varepsilon_{m}}{6 \eta} R_{e}\left[\frac{\varepsilon_{p}^{*}-\varepsilon_{m}^{*}}{\varepsilon_{p}^{*}+2 \varepsilon_{m}^{*}}\right] \nabla|E|^{2}
$$

where $\varepsilon_{m}$ and $\varepsilon_{p}$ is the relative permittivity of the medium and particle, given as $\varepsilon^{*}=$ $\varepsilon-j \sigma / \omega(\varepsilon, \sigma$, and $\omega$ being permittivity, conductivity, and angular frequency). $a$ is the particle radius, and $\eta$ is fluid viscosity. $R_{e}\left[\frac{\varepsilon_{p}^{*}-\varepsilon_{m}^{*}}{\varepsilon_{p}^{*}+2 \varepsilon_{m}^{*}}\right]$ is the real part of the Clausius-Mossotti factor (Castellanos et al., 2010). Based on Eq. 4, DEP velocity is size dependent; therefore it is not effective when enriching small molecules such as BPA. Enrichment of macromolecules would be more effective with fluid movements that carry molecules to the electrodes for binding.

For small molecule detection, enrichment by ACEO or ACET convection was found to be more effective than DEP (Cui et al., 2016, Cheng et al., 2016). ACEO (Islam et al.,2010) and the ACET effect (Wu et al., 2007) both generate circulating vortices at microelectrode surfaces to aid probe-analyte binding, whereas ACEO typically dominates at low ionic strengths and the ACET effect is more pronounced in fluids with higher ionic strength. Under the influence of an inhomogeneous AC electrical field, ACEO flow is caused by the movement of induced free ions in the double layer under the electrical field tangential to the electrode surface. Due to the viscosity between the free ions and fluid, the suspend fluid will be dragged by the motion of free ions to form microflows. A generic equation for ACEO fluid velocity is given as

$$
u_{A C E O}=-\left(\frac{\varepsilon_{m}}{\eta}\right) \cdot \Delta \xi \cdot E
$$

where $\eta$ and $\varepsilon_{m}$ are the viscosity and permittivity of the medium, $E$ is the tangential electric field and $\Delta \xi$ is the voltage drop over the charged double layer. Usually ACEO is effective for fluid manipulation in electrolytes with low electric conductivities $\left(<10^{-2} \mathrm{~S} / \mathrm{m}\right)$ in the low frequency range $(<100 \mathrm{kHz})$.

ACET velocity can be expressed as (Green et al., 2001):

$$
u_{A \mathrm{CE} T}=\frac{1}{2} R_{e}\left[\frac{\varepsilon_{m}(\alpha-\beta)}{\sigma+i \omega \varepsilon_{m}}(\nabla \mathrm{T} \cdot E) E^{*}-\frac{1}{2} \varepsilon_{m} \alpha|E|^{2} \nabla \mathrm{T}\right] l^{2} / \eta
$$


where $\mathrm{E}$ is the electric field strength, and $\mathrm{T}$ is the absolute temperature in $\mathrm{K}, \alpha=$ $\left(\frac{1}{\varepsilon_{\mathrm{m}}}\right)\left(\frac{\partial \varepsilon_{\mathrm{m}}}{\partial \mathrm{T}}\right) \sim-0.004 \mathrm{~K}^{-1}$ and $\beta=\left(\frac{1}{\sigma}\right)\left(\frac{\partial \sigma}{\partial \mathrm{T}}\right) \sim 0.02 \mathrm{~K}^{-1}$ for aqueous media. $l$ is the characteristic length of the device, typically on the order of electrode spacing. The ACET effect force could generate body force on fluid, which will in turn induce microflows.

To evaluate the effectiveness of three ACEK mechanisms on enrichment of BPA molecules, theoretical calculations based on Eqs. (4)-(6) were conducted. Table 1 shows the calculated velocities of DEP, ACEO and ACET effects on BPA molecules. (The detailed calculation process can be found in the supplementary material.) Different fluid conductivities were used for ACEO and ACET enrichment, corresponding to optimal experimental conditions. It can be seen that the ACEO velocity is much larger than the DEP and ACET velocities. Hence, the ACEO effect was used in the present work for rapid and sensitive BPA detection.

Table.1 Estimated DEP, ACET and ACEO forces.

\begin{tabular}{|c|c|c|c|}
\hline Velocity & DEP velocity & $\begin{array}{c}\text { ACET velocity } \\
(0.18 \mathrm{~S} / \mathrm{m})\end{array}$ & $\begin{array}{c}\text { ACEO velocity } \\
(0.06 \mathrm{~S} / \mathrm{m})\end{array}$ \\
\hline $\mathrm{E}=43 \mathrm{mV} / \mu m$ & $5.15 \times 10^{-11} \mathrm{~m} / \mathrm{s}$ & $4.11 \times 10^{-6} \mathrm{~m} / \mathrm{s}$ & $1.21 \times 10^{-4} \mathrm{~m} / \mathrm{s}$ \\
\hline
\end{tabular}

\section{Material and methods}

\subsection{Chemical reagents}

Buffers and sample solutions were prepared based on purified water. 4,4'Isopropylidenediphenol (bisphenol A, BPA), 4,4'-Sulfonyldiphenol (bisphenol S, BPS), 4,4'-Methylenediphenol (bisphenol F, BPF) and 6-Mercaptohexanol were purchased from Sigma-Aldrich (St. Louis, MO, USA). Dimethyl Sulfoxide (DMSO) was purchased from Fisher Scientific (Pittsburgh, PA).

A BPA aptamer specific to BPA with 5' thiol modifier C6 SH (Fisher Scientific, PA) was immobilized onto the electrodes at a concentration of $2.0 \mu \mathrm{M}$. The aptamer was incubated in a humidor at room temperature for 16 hours. The sequence of the aptamer (5' to 3') is 5' -CCG GTG GGT GGTCAG GTG GGA TAG CGT TCC GCG TAT GGC CCA GCG CAT CAC GGG TTC GCA CCA-3’. 
After aptamer immobilization, the electrode surface was blocked with 6mercaptohexanol at $1.0 \mathrm{mM}$ in purified water.

BPA, BPS and BPF were initially dissolved in DMSO to make $10 \mathrm{mM}$ stock solution followed by dilutions with DMSO to make a series of concentrations at $1.0 \mu \mathrm{M}, 100 \mathrm{nM}, 10 \mathrm{nM}, 1.0 \mathrm{nM}$ and $0.1 \mathrm{nM}$. The above concentrations were further diluted in purified water to make the working solution at $10 \mathrm{pM}, 1.0 \mathrm{pM}$, $100 \mathrm{fM}, 10 \mathrm{fM}$ and $1.0 \mathrm{fM}$ for testing. The concentrations of DMSO were kept constant in all the standards including in the background control buffer and serum samples.

Blood samples were collected from pregnant women who entered labor and delivery at the University of Tennessee Medical Center, Knoxville. All samples and clinical information were collected under Health Insurance Portability and Accountability Act (HIPAA) compliance from study participants after obtaining written informed consent under clinical research protocols approved by the University of Tennessee Institutional Review Boards (IRBs). Maternal and Cord blood were collected at the time at delivery. Serum samples were prepared after blood samples were clotted, followed by centrifugation for 15 minutes at 2,000 rpm under 4 degrees. In addition, a pooled sample from maternal and cord serum was created to use as a matrix for spiking tests. To test the matrix effect of serum, various concentrations of BPA at $1.0 \mu \mathrm{M}, 100 \mathrm{nM}, 10 \mathrm{nM}, 1 \mathrm{nM}$ and $0.1 \mathrm{nM}$ in DMSO were spiked in human serum. The spiked serum samples were further diluted 1:1000 (v/v) in purified water. The final concentrations of BPA in spiked serum samples were $10 \mathrm{pM}, 1.0 \mathrm{pM}, 100 \mathrm{fM}, 10 \mathrm{fM}$ and $1.0 \mathrm{fM}$.

\subsection{Preparation of microelectrode sensor chips}

In this work, interdigitated microelectrodes were designed for ACEK bindingacceleration and impedance measurement of serum samples. The platinum planar microelectrodes were fabricated on silicon wafers. (A microscopic image of electrodes is shown in the supplementary material.) The microelectrode arrays had interdigitated configuration with a characteristic length of $6 \mu \mathrm{m}$ (with widths of 6 $\mu m$ separated by $6 \mu m$ gaps).

The interdigitated microelectrodes were cleaned thoroughly before probe immobilization. The chips were cleaned with the following steps sequentially: soaked in acetone for 20 minutes; rinsed in isopropyl alcohol (IPA) for 30 seconds; 
rinsed in deionized water for 30 seconds; and dried with an air gun. Cleaned chips were transferred into a UV Ozone cleaner and treated for 15 minutes. A silicone microchamber $(2 \mathrm{~mm}$ in diameter and $0.9 \mathrm{~mm}$ in depth, JTR24R, GraceTM Biolabs) was sealed onto the chip with the electrodes inside for sample loading; then the chamber was cleaned with an air gun.

After pretreatment, a specific aptamer against BPA $(2 \mu \mathrm{M}$, diluted in purified water) was immobilized onto the electrode surface in a humidor for $16 \mathrm{~h}$. After immobilization, the electrodes were blocked with $1 \mathrm{mM}$ 6-mercaptohexanol in purified water for $2 \mathrm{~h}$ to inhibit non-specific binding.

\subsection{Measurement procedure}

An impedance analyzer (Agilent 4294A) was used to acquire the capacitance data that were recorded by software (Data Transfer V3.0, SEKONIC ${ }^{\circledR}$ ). A $20 \mathrm{kHz}$ AC signal of no higher than $600 \mathrm{mVrms}$ was applied to the electrode cell by the Agilent 4294A as the measuring signal. $10 \mu \mathrm{L}$ of testing standards or spiked serum samples were added to the testing chamber. Then the sensor capacitance was continuously measured at the AC signal for 30s; detection was finished when the capacitance measurement was done. The change rate of normalized capacitance was then calculated to indicate the binding level of BPA, which is shown as the slope of normalized capacitance versus time $(\% / \mathrm{min})$, found by a least square linear fitting method. The normalized capacitance was calculated as $C_{t} / C_{0}$, where $C_{t}$ and $C_{0}$ are the capacitance values at time $t$ and time zero, respectively.

\section{Results and discussions}

\subsection{Detection of BPA from analytical samples}

A series of BPA standards (1.0 fM to $10 \mathrm{pM})$ in purified water was tested to evaluate the performance of the method. An AC signal of $600 \mathrm{mVrms}$ at $20 \mathrm{kHz}$ was used to measure the sensor capacitance for 30 seconds.

Figure 2a shows representative curves of normalized capacitances for different BPA concentrations, demonstrating how $\mathrm{d} \mid \mathrm{C} / / \mathrm{dt}$ curve would change with time. During the tests, the capacitances changed linearly with time, and the capacitance change increased commensurate with increasing concentrations of 
BPA, corresponding to increasing levels of BPA binding. The slope of these capacitance curves, i.e., the normalized capacitance change rate, was found by least square linear fitting, which provides a quantitative indication of macro-molecules bound to the electrode surface. The change rates were found to be $-65.9 \% / \mathrm{min}$, $115.6 \% / \mathrm{min},-130.2 \% / \mathrm{min},-229.4 \% / \mathrm{min}$ and $-269.7 \% 0 / \mathrm{min}$ for BPA levels at 1.0 fM, $10 \mathrm{fM}, 100 \mathrm{fM}, 1.0 \mathrm{pM}$ and $10 \mathrm{pM}$, respectively.

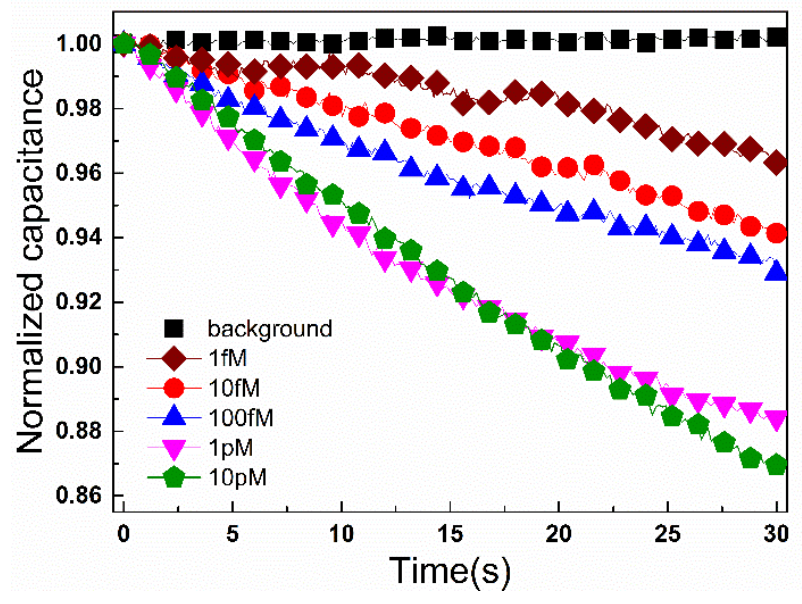

(a)

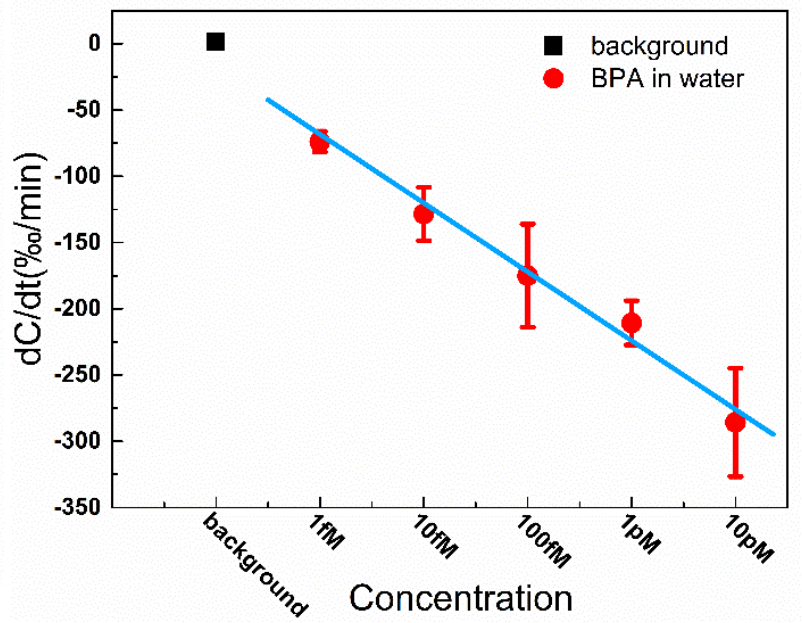

(b)

Fig. 2 Capacitance change rates in response to different concentrations of BPA (a) Normalized capacitance change as a function of time within 30 seconds for various levels of BPA spiked in water. (b) Capacitance change rate as a function of BPA concentrations in water. The AC signal used was 600 $\mathrm{mVrms}$ at $20 \mathrm{kHz}$.

The tests shown in Fig. 2a were done in triplicate. Figure $2 b$ gives the averages and standard deviations (SDs) of the sensor responses, which also shows a correlation between BPA concentrations and capacitance change rates. The BPA samples showed change rates of $-73.86 \pm 7.83 \% / \mathrm{min},-128.45 \pm 20.13 \% / \mathrm{min}$, $175.01 \pm 38.98 \% / \mathrm{min},-210.76 \pm 16.68 \% / \mathrm{min}$ and $-285.78 \pm 40.99 \% / \mathrm{min}$, for 1.0 $\mathrm{fM}$ to $10 \mathrm{pM}$ BPA, respectively (Fig. 2b). Within the range of $1.0 \mathrm{fM}$ to $10 \mathrm{pM}$ BPA, dC/dt exhibited a logarithm dependence on BPA concentration. A linear, inverse association between $\mathrm{dC} / \mathrm{dt}$ and BPA concentration was observed. The dependence is expressed as

$$
\mathrm{y}(\% \mathrm{o} / \mathrm{min})=-47.702 \log (\mathrm{BPA} \text { concentration in } \mathrm{fM})-74.323
$$

with Pearson correlation coefficient $\mathrm{R}^{2}=0.991$. With the limit of detection (LOD) defined as the concentration with a change rate more than three standard deviations 
from that of the control tests $(1.43 \pm 1.42 \% / \mathrm{min})$, the sensor had a limit of detection lower than $1.0 \mathrm{fM}(-73.86 \pm 7.83 \% / \mathrm{min})$.

To demonstrate that the ACEO-based capacitive sensing is more sensitive than ACET-based sensing, comparison experiments on BPA in phosphate buffered saline (PBS) were also conducted using $6 \mu \mathrm{m}$ electrode chips. In agreement with data shown in Table 1, ACET-based capacitive sensing yielded less sensitive results than ACEO-based sensing. The LOD for samples in PBS were between 10 $\mathrm{fM}$ and $100 \mathrm{fM}$. (See supplementary material for details of the comparison.)

\subsection{Detection of BPA from clinical serum samples}

To evaluate the performance of the sensor in a complex matrix, a series of BPA concentrations (1.0 fM to $1.0 \mathrm{pM}$ ) were spiked in 1:1,000 diluted human serum. Unspiked serum aliquot diluted with water containing the same amount of DMSO was used as the background control. For BPA-spiked serum samples, the capacitance decreased monotonically with time due to the binding reaction. As the spiked BPA level increased, the capacitance change rate became more negative, which is expected. The tests were repeated six times, and the averaged responses with error bars were plotted against BPA concentrations in Fig. 3. The change rate was $-26.91 \pm 9.42 \% / \mathrm{min}$ at $1.0 \mathrm{fM}$ of BPA, and reached almost $-207.09 \pm 9.79 \% \mathrm{~min}$ at $1.0 \mathrm{pM}$ of BPA. The dose response for BPA in diluted serum was found to be

$$
\mathrm{y}(\% \mathrm{~min})=-62.876 \log (\mathrm{BPA} \text { concentration in } \mathrm{fM})-21.237
$$

with Pearson correlation coefficient $\mathrm{R}^{2}=0.985$. The capacitances of the unspiked serum sample stayed roughly constant during the test, with a capacitance change rate of $-0.75 \pm 2.84 \% / \mathrm{min}$. Based on the background response, a cut-off value of $21.2 \%$ /min was established for $\mathrm{d}|\mathrm{C}| / \mathrm{dt}$ values. A serum sample is considered to be BPA-negative if its $\mathrm{d}|\mathrm{C}| / \mathrm{dt}$ value is more positive than $-21.2 \% / \mathrm{min}$. The limit of detection is approximately $1.0 \mathrm{fM}$ for serum based sample. 


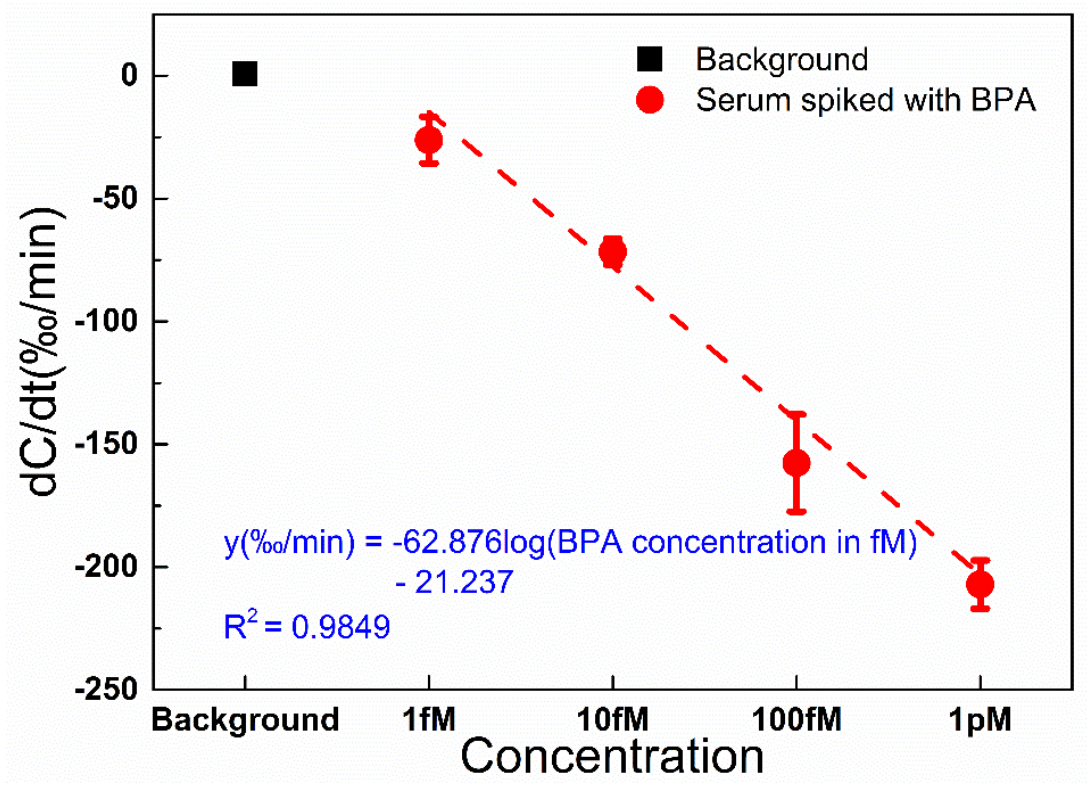

Fig. 3. Capacitance change rate for serum spiked with various concentrations of BPA (red solid circle) and background (black solid square).

\subsection{Selectivity of biosensor for BPA detection in serum samples}

To evaluate the specificity of the aptamer, various concentrations of the structurally similar molecules BPF and BPS were spiked into serum and tested for their binding against the BPA aptamer. Various concentrations of BPF and BPS samples were prepared in the same way as BPA. The capacitance change rates for $1.0 \mathrm{fM}$ to $1.0 \mathrm{pM}$ BPF and BPF were compared with those of the BPA spiked samples (Fig. 4). Based on their $\mathrm{d}|C| / \mathrm{dt}$ values, even at $1.0 \mathrm{pM}, \mathrm{BPF}$ and BPS spiked serum samples still produced negligible responses, which is less than that from 1.0 fM BPA in serum samples. These results indicate there is little, if any, cross-reactivity of BPA aptamer with BPF and BPS. All of the BPF and BPS responses fell within the cut-off values, such that the serum samples were considered to be BPA-negative. 


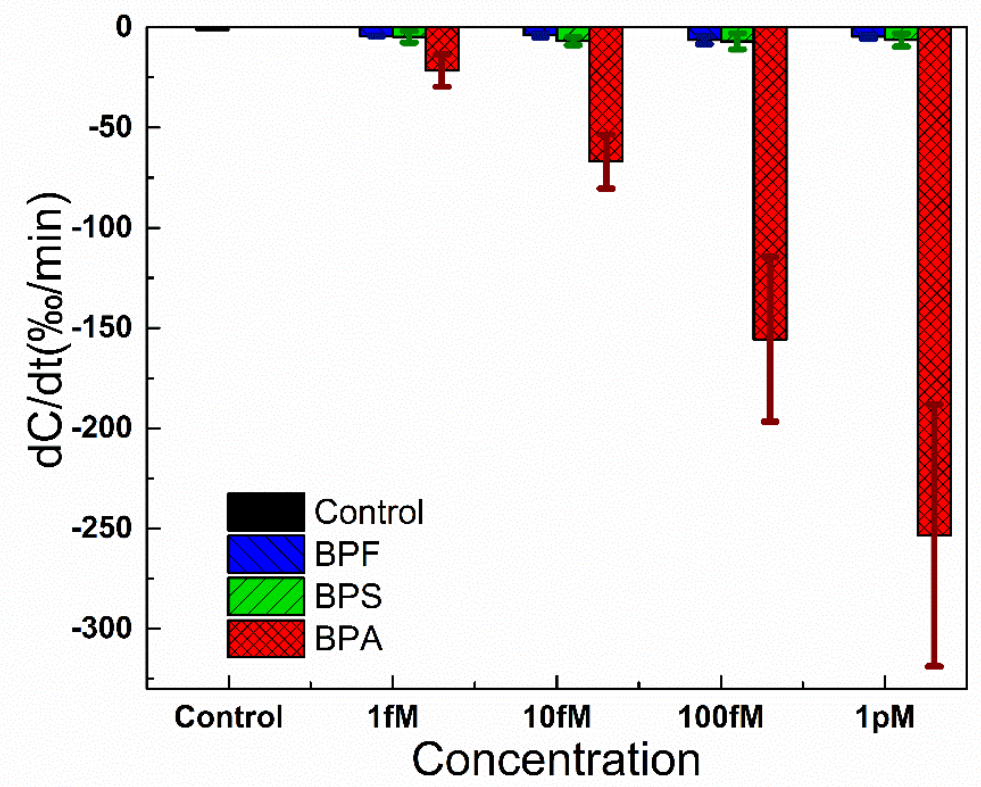

Fig. 4. Capacitance change rate for unspiked serum (dark solid square) and serum spiked with various concentrations of BPF (red solid circle), BPS (blue solid triangle) and BPA (green solid diamond).

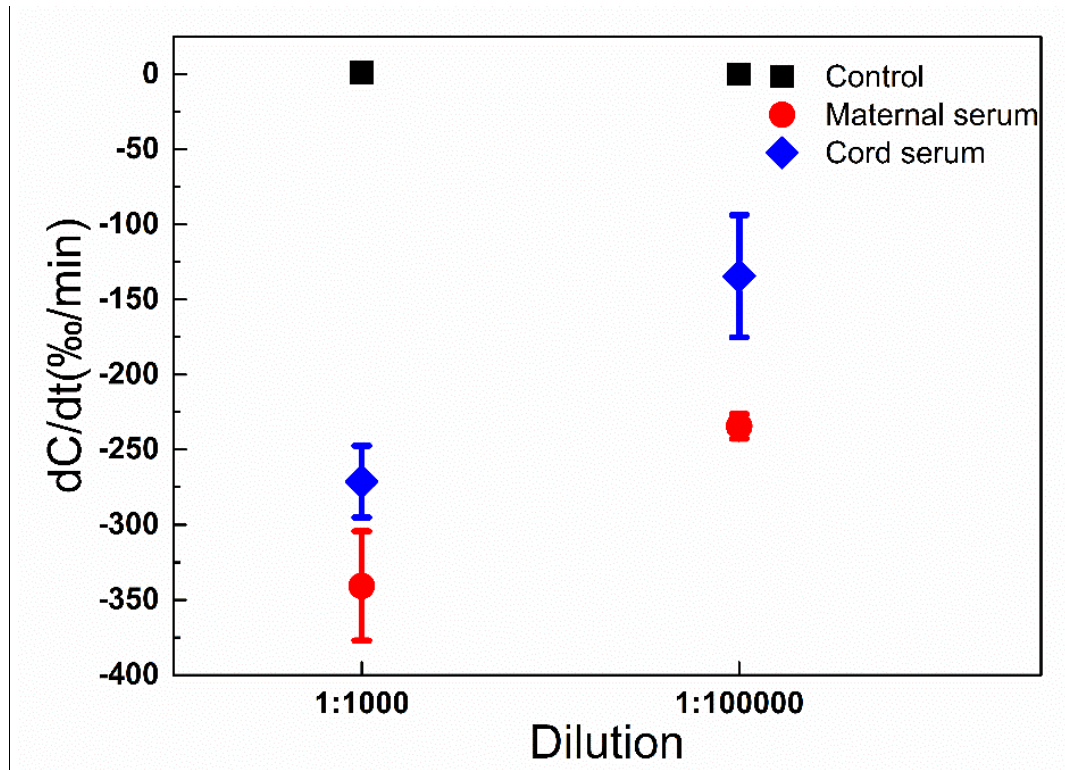

Fig. 5 Capacitance change rate for a pair of maternal and cord serum at two dilutions.

\subsection{Detection of BPA in individual maternal and cord serum samples}

Lastly, the levels of BPA in a pair of maternal and cord serum samples were prepared as described in Sec. 4.1, again with the unspiked pooled serum sample as the control. The levels of BPA in maternal and cord serum samples were tested under two different dilutions. In the first set of experiments, the sera were diluted 
at $1: 1,000$. The sensor produced a change rate of $-340.75 \pm 36.43 \% / \mathrm{min}$ for the maternal serum sample and $-271.23 \pm 23.90 \%$ /min for the cord serum sample (Fig. 5 ), indicating BPA levels well over $1.0 \mathrm{pM}$ in both samples, which were at the upper limit of sensor's range. Therefore, the serum samples were further diluted another 100-fold in $0.001 \mathrm{x}$ PBS and re-tested. The magnitude of the responses decreased accordingly: $-234.50 \pm 8.18 \% \mathrm{~min}$ for the maternal serum and $134.39 \pm 40.68 \% / \mathrm{min}$ for the cord serum, which corresponded to approximately $302 \mathrm{nM}$ and $2.2 \mathrm{nM}$ of BPA in neat maternal and cord samples, respectively. These concentrations were within the reported BPA concentration range in the literature. (Jun et al., 2016, Chou et al., 2010) When applying the sensor to the practical sample, spiked maternal and cord serum samples were clearly differentiated from the unspiked serum sample, further demonstrating the specificity and adaptability of our method.

\section{Conclusions}

In summary, we developed a capacitive aptasensor for the rapid and label-free detection of BPA in biological matrices. Planar platinum electrodes were immobilized with an aptamer bound specifically to BPA. When samples containing BPA were loaded to the sensor, interfacial capacitance decreased due to the binding of BPA to the aptamer. Using ACEK convection, we were able to detect BPA within 30 seconds after the sample was loaded on the chip. The sensor had a LOD of $1.0 \mathrm{fM}$, which is several orders of magnitude more sensitive than those reported by current detection methods such as HPLC (LOD is $10^{-6} \mathrm{M} \sim 10^{-9} \mathrm{M}$ level) (Gallardo et al., 2016; Yang et al., 2016) or ELISA (LOD is $10^{-9} \mathrm{M} \sim 10^{-10} \mathrm{M}$ level)( Moreno et al., 2011; Yang et al., 2016). Further development of this sensor may yield an effective platform for BPA detection in biological matrices as well as in environmental samples.

\section{Acknowledgements}

Microfabrication was conducted at the Center for Nanophase Materials Sciences, which is sponsored at Oak Ridge National Laboratory by the Scientific User Facilities Division, US Department of Energy. The work was supported by the University of Tennessee's Initiative for PON/POC Nanobiosensing, and the Center for Wildlife Health Organized Research Unit. X. Lin acknowledges the 
support of the National Natural Science Foundation of China (Project No. 61377001) and the Fundamental Research Funds for the Central Universities (Project No.CDJZR12125501 and Project no. 10611201312011). 


\section{References}

A.M. Rocha, Q. Yuan, D. Close, K.B. O’dell, J. Fortney, J. Wu, and T.C. Hazen, 2016, Biosensors and Bioelectronics. 85, 915-923.

Ashley-Martin, J., Dodds, L., Levy, A.R., Platt, R.W., Marshall, J.S., Arbuckle, T.E., 2015. Environ Res $140,360-368$.

Avila, M., Zougagh, M., Ríos, Á., 2008. Trends Anal. Chem 27, 54-65.

Barker, D.J., Eriksson, J.G., Forsén, T., Osmond, C., 2002. Int J Epidemiol 31(6), 1235-1239.

Bhandari, R.K., Deem, S.L., Holliday, D.K., Jandegian, C.M., Kassotis, C.D., Nagel, S.C., Tillitt, D.E., Vom Saal, F.S., Rosenfeld, C.S., 2015. Gen Comp Endocrinol 214,195-219.

Birceanu, O., Mai, T., Vijayan, M.M., 2015. Aquat Toxicol 168, 11-18.

Castellanos, A., Ramos, A., Gonzalez, A., Green, N. G., Morgan, H., 2003. Journal of Physics D: Applied Physics 36(20), 2584-2597.

C. Cheng, S. Wang, J. Wu, Y. Yu, R. Li, S. Eda, J. Chen, G. Feng, B. Lawrie and A. Hu, 2016, ACS Appl. Mater. Interfaces 8 (28), 17784-17792

Chen, J., Xiao, Y., Gai, Z., Li, R., Zhu, Z., Bai, C., Tanguay, R.L., Xu, X., Huang, C., Dong, Q., 2015. Aquatic Toxicol 169, 204-214.

Chevalier, N., Brucker-Davis, F., Lahlou, N., Coquillard, P., Pugeat, M., Pacini, P., Panaïa-Ferrari, P., Wagner-Mahler, K., Fénichel, P., 2015. Hum Reprod 30(2), 447-453.

Chou, W.C., Chen, J.L., Lin, C.F., Chen, Y.C., Shih, F.C., Chuang, C.Y., 2010. Environ Health 10(1):110 .

Cruz, G., Foster, W., Paredes, A., Yi, K.D., Uzumcu, M., 2014. J Neuroendocrinol 26(9), 613-624.

Cui, H., Li, S., Yuan, Q., Wadhwa, A., Eda, S., Chambers, M., Ashford, R., Jiang, H., Wu, J., 2013. Analyst 138(23), 7188-7196.

Cui, H., Cheng, C., Lin, X., Wu, J., Chen, J., Eda, S., Yuan, Q., 2016. Sensors and Actuators B: Chemical $226,245-253$.

Cui, H., Wu, J., Eda, S., Chen, J., Chen, W., Zheng, L., 2015. Microchimica Acta 182(13-14), 2361-2367.

Deceuninck, Y., Bichon, E., Marchand, P., Boquien, C.Y., Legrand, A., Boscher, C., Antignac, J.P., Le Bizec, B., 2015. Anal Bioanal Chem 407(9), 2485-97.

D’Antuono, A., Dall'Orto, V.C., Balbo, A.L., Sobral, S., Rezzano, I., 2001. Journal of Agricultural and Food Chemistry 49(3), 1098-1101.

Falck, A.J., Mooney, S., Kapoor, S.S., White, K.M., Bearer, C., El Metwally, D., 2015. Pediatric Clinics of North America 62(5), 1173-1197.

Fenichel, P., Chevalier, N., Brucker-Davis, F., 2013. Ann Endocrinol (Paris) 74(3), 211-220.

Fernández, M.F., Arrebola, J.P., Jiménez-Díaz, I., Sáenz, J.M., Molina-Molina, J.M., Ballesteros, O., Kortenkamp, A., Olea, N., 2015. Reprod Toxicol 59, 89-95. 
Gallardo, E. M. R., Lucena, R., Cárdenas, S., Valcárcel, M. 2016. Microchemical Journal, 124, 751-756.

Green, N.G., Ramos, A., González, A., Castellanos, A., Morgan, H., 2001. Journal of Electrostatics 53, 71-87.

Hart, R., Lec, R., Noh, H., 2010. Sensors and Actuators B: Chemical 147(1), 366-375.

He Y., Miao M., Herrinton L.J., Wu C., Yuan W., Zhou Z., Li D.K., 2009. Environmental Research 109(5), 629-633.

Hegnerová, K., Piliarik, M., Šteinbachová, M., Flegelová, Z., Černohorská, H., Homola, J., 2010. Anal Bioanal Chem 398, 1963-1966.

Inoue, K., Kato, K., Yoshimura, Y., Makino, T., Nakazawa, H., 2000. J Chromatogr B Biomed Sci Appl 749(1), 17-23.

Islam, N., Lian, M., \& Wu, J., 2007. Microfluidics and Nanofluidics 3(3), 369-375.

Jun, Y., Machiko, M., Seiko, S., Atsuko, A., Chihiro, M., Toru, M., Reiko, K., 2016. Chemosphere, 164, $25-31$.

Kim, A., Lee, C.R., Jin, C.F., Lee, K.W., Lee, S.H., Shon, K.J., Park, N.G., Kim, D.K., Kang, S.W., Shim, Y.B., Park, J.S., 2007. Chemosphere 68, 1204-1209.

Lee, J., Jo, M., Kim, T.H., Ahn, J., Lee, D., Kim, S., Hong, S., 2010. Lab on A Chip 11(1), 52-56.

Leclerc, F., Dubois, M.F., Aris, A., 2014. Hypertens Pregnancy 33(3), 341-348.

Li, S., Cui, H., Yuan, Q., Wu, J., Wadhwa, A., Eda, S., Jiang, H., 2014. Biosensors and Bioelectronics 51(2), 437-443.

Liao, S.L., Tsai, M.H., Lai, S.H., Yao, T.C., Hua, M.C., Yeh, K.W., Chiang, C.H., Huang, S.Y., Huang, J.L., 2016. Pediatric Research 79,438-444.

Meesters, R.J.W., Schroder, H.F., 2002. Analytical chemistry 74(14), 3566-3574.

Mikołajewska, K., Stragierowicz, J., Gromadzińska, J., 2015. Int J Occup Med Environ Health 28(2), 209-241.

Moreno, M. J., D'Arienzo, P., Manclús, J. J., Montoya, A., 2011. Journal of Environmental Science \& Health Part B Pesticides Food Contaminants \& Agricultural Wastes, 46(6), 509-17.

Nahar, M.S., Liao, C., Kannan, K., Harris, C., Dolinoy, D.C., 2015. Chemosphere 124, 54-60.

Nambiar, S., Yeow, J.T.W., 2011. Biosensors and Bioelectronics 26 (5), 1825-1832.

Ndlovu, T., Arotiba, O.A., Sampath, S., Krause R.W., Mamba B.B., 2012. Sensor 12(9), 11601-11611.

Ntsendwana, B., Mamba, B.B., Sampath, S., Arotiba, O.A., 2012. Int. J. Electrochem. Sci. 7(4), 35013512.

Ragavan, K. V., Rastogi, N. K., \& Thakur, M. S., 2013. TrAC Trends in Analytical Chemistry 52(2), 248260.

Rochester, J.R., 2013. Reprod Toxicol 42(12), 132-155. 
Rochester, J.R., Bolden, A.L., 2015. Environ Health Perspect 123(7), 643-650.

Sajiki, J., Hasegawa, Y., Hashimoto, H., Makabe, Y., Miyamoto, F., Yanagibori, R., Shin, J., Shimidzu, Y., Morigami, T., 2008. Toxicol Mech Methods 18(9), 733-738.

Seachrist, D.D., Bonk, K.W., Ho, S.M., Prins, G.S., Soto, A.M., Keri, R.A., 2015. Reprod Toxicol 59, 167-182.

Suvorov, A., Waxman, D.J., 2015. Reprod Toxicol 57, 59-72.

S. Li, Y. Ren, H. Cui, Q. Yuan, J. Wu, S. Eda, H. Jiang,2015, Electrophoresis, 36(3), 471-474.

Troisi, J., Mikelson, C., Richards, S., Symes, S., Adair, D., Zullo, F., Guida, M., 2014. Placenta 35(11), 947-952.

Tu, X., Yan, L., Luo, X., Luo, S., Xie, Q., 2009. Electroanalysis 21(22), 2491-2494.

Varayoud, J., Ramos, J.G., Muñoz-de-Toro, M., Luque, E.H., 2014. Vitam Horm 94, 253-275.

Vashist, S.K., Zheng, D., Al-Rubeaan, K., Luong, J.H.T., Sheu, F., 2011. Biotechnol. Adv. 29(2), 169188.

Walter, J.G., Stahl, F., Scheper, T., 2012. Eng. Life Sci. 12(5), 496-506.

Wu, J., Lian, M., Yang, K., 2007. Applied physics letters 90(23), 234103-234103-3.

Yang, F., Xu, L., Zhu, L., Zhang, Y., Meng, W., Liu, R. 2016.Environmental Science and Pollution Research, 23(11), 10714-10721.

Yang, K., Wu, J., 2010. Biomicrofluidics 4(3), 3315-3325.

Yang, W., Ratinac, K.R., Ringer, S.P., Thordarson, P., Gooding, J.J., Braet, F., 2010. Angew Chem. Int. Ed. 49 (12) 2114-2138.

Ye, Z., Zhou, C., Yan, X., Yan, Y., Wang, Q., 2015. Analytica Chimica Acta 883:81-89.

Yin, H., Zhou, Y., Ai, S., Chen, Q., Zhu, X., Liu, X., Zhu, L., 2010. J. Hazard. Mater. 17(1-3), 236-243.

Yin, H., Zhou, Y., Ai, S., 2009. J. Electroanal. Chem. 626(1-2), 80-88.

Yoon, K., Kwack, S.J., Kim, H.S., Lee, B.M., 2014. J Toxicol Environ Health B Crit Rev. 17(3), 127-174. 